\title{
11. ¿Cómo realizar el seguimiento de los pacientes con fibrosis pulmonar idiopática?
}

\author{
HUGO VALENZUELA C.*, MIRTHA REYES H.**, CLAUDIA PINO V.*** y MAURICIO TAPIA G.****
}

\section{How to monitor patients with idiopathic pulmonary fibrosis?}

Idiopathic pulmonary fibrosis (IPF) has been classified as mild or early - moderate - severe or advanced disease, with no cut-off points in clinical, functional or imaging parameters. There is no consensus yet on which is the main parameter to be measured although the functional variables such as forced vital capacity $(F V C)$, carbon monoxide diffusion capacity $\left(D L_{C O}\right)$ and 6-minute walk test, have been routinely used in clinical practice and in the main clinical studies of treatment, are often not representative of the clinical evolution. Therefore, composite indices or scores such as the GAP (Gender-Age-Physiology) scale have been developed that could be useful in the follow-up of patients.

Key words: Idiopathic pulmonary fibrosis; Follow-Up of Studies; Vital Capacity; Walk test.

\section{Resumen}

La fibrosis pulmonar idiopática (FPI) se ha clasificado en enfermedad leve o temprana-moderadasevera o Avanzada, sin puntos de corte en parámetros clínicos, funcionales o imagenológicos. No existe aún consenso en cual es el principal parámetro que se debe medir. Si bien las variables funcionales como la capacidad vital forzada (CVF), capacidad de difusión de monóxido de carbono $\left(D L_{C O}\right)$ y test de caminata de 6 minutos se han utilizado de forma rutinaria en la practica clínica y en los principales estudios clínicos de tratamiento muchas veces no son representativos de la evolución clínica. Por lo anterior se han desarrollado, índices o puntajes compuestos como la escala GAP (Gender-AgePhysiology) que podrían ser útiles en el seguimiento de los pacientes.

Palabras clave: Fibrosis pulmonar idiopática; Estudios de Seguimiento; Capacidad Vital; Prueba de Caminata.

Tradicionalmente la fibrosis pulmonar idiopática (FPI) se ha clasificado en enfermedad leve o temprana-moderada-severa o Avanzada, sin puntos de corte en parámetros clínicos, funcionales o imagenológicos. La ausencia de herramientas validadas o adoptadas en general son la manifestación de falta de consenso en esta área.

\section{¿Porque etapificar al paciente?}

- Entrega de información pronóstica a paciente y a su familia.
- Identificar tendencias para el inicio de diferentes tratamientos, como fármacos antifibróticos, oxígeno o derivación a trasplante, sobretodo en lugares con recursos limitados.

- Protocolos-unificar criterios.

Sabemos que, observando su evolución en el tiempo, podemos tener distintas velocidades de progresión (Figura 1). Puede tener un curso rápido desde su inicio, puede tener una o varias exacerbaciones que aceleren el deterioro funcional y lleven a insuficiencia respiratoria o bien tener una evolución lenta través de varios años ${ }^{1}$.

* Instituto Nacional del Tórax y Clínica Alemana de Santiago. Santiago, Chile.

** Hospital Carlos Van Buren, Médico Ad Honorem de Cátedra de Medicina Interna, Universidad de Valparaíso. Valparaíso, Chile.

*** Hospital de Carabineros e Instituto Nacional del Tórax. Santiago, Chile.

**** Hospital de Coyhaique. Coyhaique, Chile. 


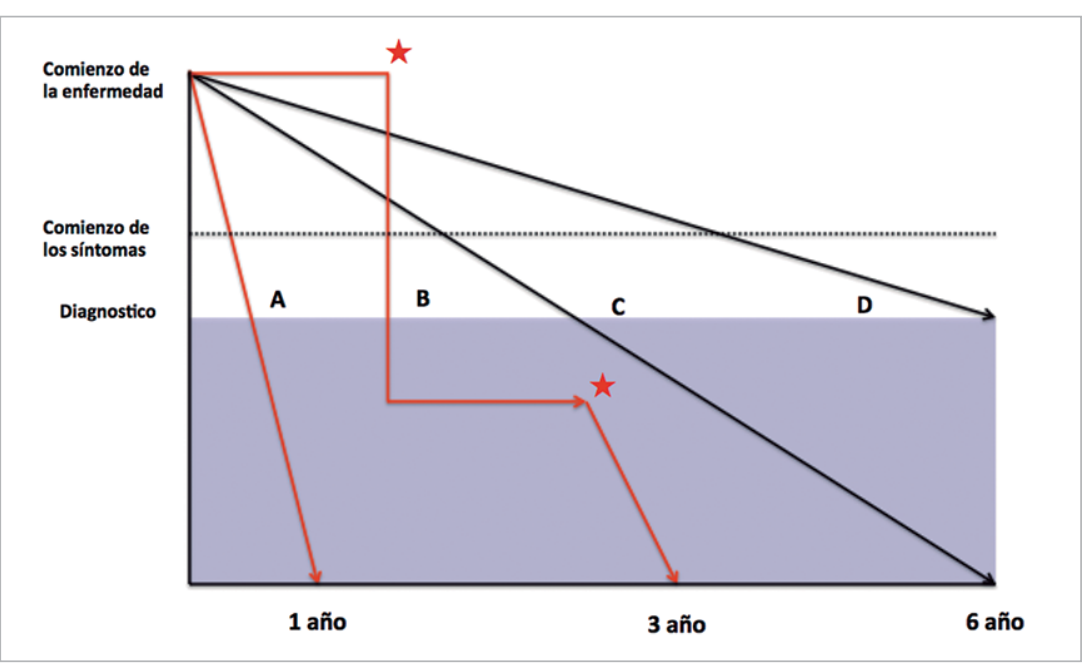

Figura 1. Representación esquemática de posibles cursos clínicos de pacientes con fibrosis pulmonar idiopática. La tasa de disminución y progresión a la muerte puede ser rápida (línea $\mathrm{A}$ ), lenta (líneas C y D) o mixta (curva B), con períodos de estabilidad y exacerbaciones (estrella). Gráfico adaptado de Ley, et $\mathrm{al}^{1}$.
No existe aún consenso en cual es el principal parámetro que medir para los trabajos clínicos en FPI. Se han utilizado, por ejemplo, tasas de hospitalización, tasas de exacerbaciones, parámetros funcionales como capacidad vital forzada (CVF), capacidad de difusión de monóxido de carbono $\left(\mathrm{DL}_{\mathrm{CO}}\right)$ y test de caminata de $6 \mathrm{~min}$. Se han desarrollado por otro lado, ante la falta de un parámetro ideal, índices o puntajes compuestos que han demostrado ser útiles en el seguimiento de estos pacientes.

A continuación, discutiremos los principales parámetros utilizados tanto en la práctica clínica como en los trabajos de investigación en FPI.

\section{Disnea}

Casi todos los pacientes con FPI experimentan una disnea progresiva que produce un gran impacto en su calidad de vida. La presencia de disnea y el cambio en su intensidad son un predictor independiente de supervivencia.

Para evaluar la disnea en FPI se recomienda utilizar la escala de mMRC (Medical Research Council modificada) que, si bien no fue diseñada para enfermedades pulmonares intersticiales, se ha utilizado en la estratificación de los pacientes con FPI y como predictor de progresión en mediciones longitudinales ${ }^{2}$. Por otro lado, es una herramienta de bajo costo y simple que puede ser utilizada en cada control clínico del paciente.

\section{Tos}

También se ha relacionado con progresión y pronóstico en esta enfermedad. Su evaluación no es rutinaria ni se ha estandarizado en las guías actuales, pero sí existen cuestionarios para su evaluación como el Leicester Cough Questionnaire que ha demostrado ser sensible a cambios en la progresión de la enfermedad. Se considera una herramienta para evaluar resultados en trabajos de FPI. No se utiliza en la práctica clínica diaria.

\section{Pruebas de función pulmonar}

Los estudios funcionales pulmonares son sencillos, ampliamente disponibles y por lo tanto fáciles de obtener durante las visitas de seguimiento de rutina de pacientes con $\mathrm{FPI}^{3}$.

La reproducibilidad de la espirometría, especialmente la CVF, es generalmente buena ya que el $90 \%$ de los pacientes puede repetir la prueba con un $5 \%$ de variación ${ }^{4}$. La variabilidad de $\mathrm{DL}_{\mathrm{CO}}$ es mayor. Un cambio anual absoluto en la $\mathrm{CVF}$ mayor a $10 \%$ y/o un cambio de $\mathrm{DL}_{\mathrm{CO}}$ del $15 \%$ son marcadores de progresión de enfermedad 5 . Por lo anterior, parece ser apropiado monitorear el curso de la enfermedad con CVF y $\mathrm{DL}_{\mathrm{CO}}$ a intervalos de 3 a 6 meses. Un subgrupo de pacientes con progresión rápida o empeoramiento agudo pueden no ser identificados en plazos largos de control $^{6}$. El intervalo de tiempo óptimo para la repetición de $\mathrm{CVF}$ y $\mathrm{DL}_{\mathrm{CO}}$ no se ha investigado formalmente, por lo que un enfoque flexible de monitoreo de progresión podría ser útil para algunos pacientes.

\section{Escalas compuestas}

Estos sistemas de puntuación compuesta incluyen evaluaciones clínicas, radiológicas y fisiológicas para desarrollar un modelo de predicción integrado, que es probable que sea más preciso que cualquier prueba individual ${ }^{7}$. 


\section{Índice fisiológico compuesto (IFC, CPI en por sus siglas en inglés)}

Wells et al desarrollaron el IFC, basado en el grado de enfermedad en la tomografía de tórax, con un modelo de regresión multivariado con variables fisiológica y concluyeron se correlaciona más fielmente con la mortalidad que cualquier índice de función pulmonar individual ${ }^{7}$.

\section{Índice de GAP (Gender-Age-Physiology)}

Ley et al. ${ }^{8}$ crearon este índice que es una regla de predicción clínica de mortalidad, basado en etapificar en tres grupos de riesgo, basado en variables medidas en tres años consecutivos. Así la mortalidad en grupo de bajo riesgo a los 3 años es de $16 \%$ y en el grupo de alto riesgo es de $77 \%$. El índice de GAP se realiza entonces al inicio de la evaluación del paciente y luego anualmente (Tabla 1).

\section{Estrategia de riesgo (ROSE)}

Mura et al desarrollaron el sistema de puntuación ROSE, que considera disnea (mMRC), test de caminata de 6 min (TC6M) e IFC para determinar la supervivencia a 3 años. ROSE predijo la mortalidad a 3 años con sensibilidad de 39\% y especificidad de $100 \%{ }^{9}$. Además, en cuanto a la progresión de categorías, el ROSE inicial y a los 6 meses fue predictor de mortalidad a 3 años (sensibilidad de $94 \%$ y especificidad de $40 \%$ ). Por lo anterior se puede sugerir seguimiento con ROSE inicial, 6 meses y anual por 3 años.

El índice multidisciplinario ideal o sistema de estratificación tendrá que ser simple de calcular, incluir parámetros que son fáciles de medir, seguros y validados. Este sistema multidimensional aún no se ha definido y aún debe ser incorporado en las directrices de consenso de FPI.

Por su simpleza y experiencia en su uso recomendamos el uso de escala GAP al momento del diagnóstico del paciente y en forma anual.

\section{Evolución diagnóstica y seguimiento con tomografía computada da alta resolución (TCAR)}

Estudios de seguimiento con TCAR y análisis multivariado solo demuestran asociación entre la velocidad de progresión y la extensión de la fibrosis en el TC basal, incluso casos con patrón de posible neumonía intersticial usual en TC siguen curso similar a los que tenían patrón NIU definitivo (incluso con NIU histológica). En estos casos el desarrollo de panal no se asocia a diferencias en pronóstico ${ }^{10}$.

Por lo tanto, se recomienda el uso de TCAR de tórax en el momento del diagnóstico y fren-
Tabla 1. Escala GAP

\begin{tabular}{|c|c|c|}
\hline $\begin{array}{l}\text { Parámetro } \\
\text { Género }\end{array}$ & Hombre & $\begin{array}{c}\text { Puntos } \\
1\end{array}$ \\
\hline Edad & $\begin{array}{l}61-65 \\
>65\end{array}$ & $\begin{array}{l}1 \\
2\end{array}$ \\
\hline Fisiología & $\begin{array}{l}\text { CVF } 50-75 \% \text { pred* } \\
<50 \% \text { pred } \\
\text { DL }_{\text {CO }} 36-55 \% \text { pred } \\
<36 \% \text { pred } \\
\text { No pudo }\end{array}$ & $\begin{array}{l}1 \\
2 \\
1 \\
2 \\
3\end{array}$ \\
\hline Total & $\begin{array}{l}\text { 0-3: Etapa I } \\
\text { 4-5: Etapa II } \\
\text { 6-8 puntos: Etapa III }\end{array}$ & $\begin{array}{l}\text { Mortalidad } 3 \text { años: } \\
16 \% \\
\text { Mortalidad } 3 \text { años: } \\
42 \% \\
\text { Mortalidad } 3 \text { años: } \\
\quad 77 \%\end{array}$ \\
\hline
\end{tabular}

*\% del valor normal predeterminado.

te a exacerbaciones agudas o franco deterioro funcional en el seguimiento. No se recomienda como un parámetro de seguimiento clínico para la evaluación de la progresión de la FPI, sino más bien como seguimiento anual de pesquisa de cáncer pulmonar.

\section{Bibliografía}

1.- LEY B, COLLARD HR, KING TE Jr. Clinical course and prediction of survival in idiopathic pulmonary fibrosis. Am J Respir Crit Care Med 2011; 183: 431-40.

2.- ROBBIE H, DACCORD C, CHUA F, DEVERAJ A. Evaluating disease severity in idiopathic pulmonary fibrosis. Eur Respir Rev 2017; 26: 170051.

3.- MILGER K, BEHR J. Idiopathic pulmonary fibrosis: ERS Monography. Chapter 9: Monitoring.

4.- ENRIGHT PI, BECK KC, SCHERRILL DI. Repeatability of spirometry in 18000 adults patients. Am J Respir Crit Care Med 2004; 169: 235-8.

5.- COLLARD HR, KING TE JR, BARTELSON BB, VOURLEKIS JS, SCHWARZ MI, BROWN KK. Changes in clinical and physiologic variables predict survival in idiophatic pulmonary fibrosis. Am J Respir Crit Care Med 2003; 168: 538-32.

6.- LEY B, RYERSON CJ, VITTINGHOFF E, RYU JH, TOMASSETTI S, LEE JS, et al. A multidimensional index and staging system for idiopathic pulmonary fibrosis. Ann Intern Med 2012; 156: 684-91.

7.- WELLS AU, DESAI SR, RUBENS MB, GOH NS, CRAMER D, NICHOLSON AG, et al. Idiopathic pulmonary fibrosis: a composite physiologic index derived from disease extent observed by computed tomography. Am J Respir Crit Care Med 2003; 167: 962-9.

8.- O'CONNELL OJ, EGAN JJ. Idiopathic pulmonary 
fibrosis: ERS Monography. Chapter 8: Evaluation of diesase.

9.- MURA M, PORRETTA MA, BARGAGLI E, SERGIACOMI G, ZOMPATORI M, SVERZELLATI N, et al. Predicting survival in newly diagnosed idiopathic pulmonary fibrosis: a 3-year prospective study. Eur
Respir J 2012; 40: 101-9.

10.- BROWNELL R, MOUA T, HENRY TS, ELICKER BM, WHITE D, VITTINGHOF E, et al. The use of pretest probability increases the value of high-resolution CT in diagnosing usual interstitial pneumonia. Thorax 2017; 72: 424-9.

Correspondencia a:

Dr. Hugo Valenzuela C.

Email: hvalenzuela.correa@gmail.com 